\title{
A comparison of BeadChip and WGS genotyping outputs using partial validation by sanger sequencing
}

\author{
Kirill A. Danilov ${ }^{1,2^{*}}$, Dimitri A. Nikogosov' ${ }^{1}$, Sergey V. Musienko ${ }^{1}$ and Ancha V. Baranova ${ }^{3,4}$
}

From 11th International Young Scientists School "Systems Biology and Bioinformatics" - SBB-2019

Novosibirsk, Russia. 24-28 June 2019

\begin{abstract}
Background: Head-to-head comparison of BeadChip and WGS/WES genotyping techniques for their precision is far from straightforward. A tool for validation of high-throughput genotyping calls such as Sanger sequencing is neither scalable nor practical for large-scale DNA processing. Here we report a cross-validation analysis of genotyping calls obtained via Illumina GSA BeadChip and WGS (Illumina HiSeq X Ten) techniques.

Results: When compared to each other, the average precision and accuracy of BeadChip and WGS genotyping techniques exceeded 0.991 and 0.997 , respectively. The average fraction of discordant variants for both platforms was found to be $0.639 \%$. A sliding window approach was utilized to explore genomic regions not exceeding 500 bp encompassing a maximal amount of discordant variants for further validation by Sanger sequencing. Notably, 12 variants out of 26 located within eight identified regions were consistently discordant in related calls made by WGS and BeadChip. When Sanger sequenced, a total of 16 of these genotypes were successfully resolved, indicating that a precision of WGS and BeadChip genotyping for this genotype subset was at 0.81 and 0.5 , respectively, with accuracy values of 0.87 and 0.61 .
\end{abstract}

Conclusions: We conclude that WGS genotype calling exhibits higher overall precision within the selected variety of discordantly genotyped variants, though the amount of validated variants remained insufficient.

Keywords: WGS, WES, Whole genome sequencing, Microarray genotyping, Genotype concordance, Sanger sequencing

\section{Background}

Both Whole Genome (WGS) and Whole Exome sequencing (WES) are now used in multiple avenues of clinical and scientific inquiry. Despite increased availability of these techniques and rapid decline of associated costs,

\footnotetext{
* Correspondence: danilov@atlasbiomed.com

'Atlas Biomed Group Limited, Tintagel House, 92 Albert Embankment, Lambeth, London SE1 7TY, UK

${ }^{2}$ Skolkovo Institute of Science and Technology, Bolshoy Boulevard 30, bld. 1, 121205 Moscow, Russia

Full list of author information is available at the end of the article
}

their context-dependent per-base performance remained in question. The performance characteristics of WGS/ WES include accuracy (the extent of agreement between the reference and the assay-derived nucleic sequence), precision which is broadly defined as repeatability for within-run precision and reproducibility for betweenrun precision as well as analytical sensitivity, specificity and a reportable range of the reference genome coverage [1]. While the repeatability issues were extensively presented in detail previously [2], the absence of scalable

C C The Author(s). 2020 Open Access This article is licensed under a Creative Commons Attribution 4.0 International License, which permits use, sharing, adaptation, distribution and reproduction in any medium or format, as long as you give appropriate credit to the original author(s) and the source, provide a link to the Creative Commons licence, and indicate if changes were made. The images or other third party material in this article are included in the article's Creative Commons licence, unless indicated otherwise in a credit line to the material. If material is not included in the article's Creative Commons licence and your intended use is not permitted by statutory regulation or exceeds the permitted use, you will need to obtain permission directly from the copyright holder. To view a copy of this licence, visit http://creativecommons.org/licenses/by/4.0/ The Creative Commons Public Domain Dedication waiver (http://creativecommons.org/publicdomain/zero/1.0/) applies to the data made available in this article, unless otherwise stated in a credit line to the data. 
non-NGS (next-generation sequencing) techniques for genotype calling limits evaluations of accuracy.

BeadChip genotyping is an efficient and scalable way of genotype resolution, with two inherent limitations: a necessity to decompose binary alleles and confinement to a predefined list of genotyped variants. These two limitations, however, do not prevent its usefulness for a variety of clinical and non-clinical applications [3]. Predefined nature of the variants to be tested makes BeadChip genotyping amenable to validation by either PCR (polymerase chain reaction), or Sanger sequencing which has been recently shown to have limited utility and erroneous behaviour in the validation of NGS variants [4]. A comparison of genotyping calls made by BeadChip and WGS/WES techniques may provide an insight into the possible nature of the discordant calls observed during genotyping quality control stage. Here we attempted to identify analytical issues leading to the discordance in genotyping calls made independently by WGS and BeadChip techniques.

\section{Results}

\section{Sequencing statistics}

Table 1 lists the sequencing statistics for the three sequenced samples. FastQC reports are available as Additional files 1, 2, 3, 4, 5 and 6 for sample_001, sample_ 002, sample_003, respectively. Percentage of reads falling into a category with averaged Phred scaled sequencing quality above 30 is shown by $\% \mathrm{Q}$. The absence of unpaired reads, repeatability in terms of GC content and more than $90 \%$ of bases exceeding sequencing quality of 30 was used as a mark of confidence in data quality.

\section{Mapping statistics}

All the data produced by WGS were analyzed for their depth (DOC) and breadth (BOC) of coverage using GATK 3.8 DepthOfCoverage tool [5]. The mean filtered coverage for all three samples exceeded 27x (Fig. 1a-c, Table 2), which complied with recommendations [6]. Repeatability in BOC values for each base quality interval (Fig. 1b, Table 2) and other sequencing metrics (Table 1 ) proved the quality of the sequencing.

\section{Concordance metrics}

Percentages of discordant calls per chromosome for each sample are shown in Fig. 2. The average fraction of discordant results among all comparable genotyping results (intersection of BeadChip and WGS obtained genotyping data) was at $0.3317 \%$ for sample_001 (male), $0.8448 \%$ for sample_002 (female) and 0.7392\% for sample_003 (male) which falls within the range reported previously [2]. Both the sequencing quality and mapping quality parameters were reproduced for all three sequenced samples with an average genotype concordance estimate being higher than $99 \%$.

\section{Mapping of analyzed variants}

For each pair of BeadChip-genotyped neighboring variants, distance intervals were extracted within both concordant and discordant group to generate pairs of distance values before and after each variant, followed by their visualization. The resulting maps with a Gaussian kernel density estimation are shown in Fig. 3. The observed approximate evenness of distribution for both concordant and discordant variants supported by the visible clusters allocation along the bisectrix of the axes shows that both concordant and discordant variants are evenly distributed across the chromosome length, with no congregation across the genome. The only observed difference in cluster allocation arises from the frequency of observation for the variants from each group. In other words, less frequent discordant group corresponds to lower mapping density and, consequently, to more considerable distances between every two neighboring variants.

Overall randomness of the locations of discordant genotypes across the genome, measured as cluster evenness and cluster center distance from the bisectrix, was high. However, because locations of discordant variants were limited to variants present on the corresponding BeadChip, the comparison with WGS was necessarily limited to the locations of BeadChip genome variants only. For this reason, patterns in discordant genotypes location throughout the genome were only detectable at locations thoroughly covered by variants presented at BeadChip, which generally are outside any complex

Table 1 Raw sequencing data statistics summary

\begin{tabular}{llllllll}
\hline Sample & Read orientation & Mean read quality (Phred score) & Number of reads & $\%$ GC & $\%$ Q > 30 & Number of bases (1e6) & Mean read length (bp) \\
\hline 001 & R1 & 39.31 & 399036358 & 41.07 & 95.56 & 59855.45 & 150.0 \\
& R2 & 36.76 & 399036358 & 41.21 & 86.17 & 59855.45 & 150.1 \\
002 & R1 & 39.28 & 375900168 & 41.06 & 95.47 & 56385.03 & 150.2 \\
& R2 & 36.8 & 375900168 & 41.22 & 86.32 & 56385.03 & 150.3 \\
& R1 & 39.32 & 385826012 & 40.95 & 95.59 & 57873.9 & 150.4 \\
& R2 & 36.49 & 385826012 & 41.08 & 85.15 & 57873.9 & 150.5 \\
\hline
\end{tabular}

The table describes the raw sequencing data quality metrics for all sequenced samples; GC Guanine-cytosine sequence content 

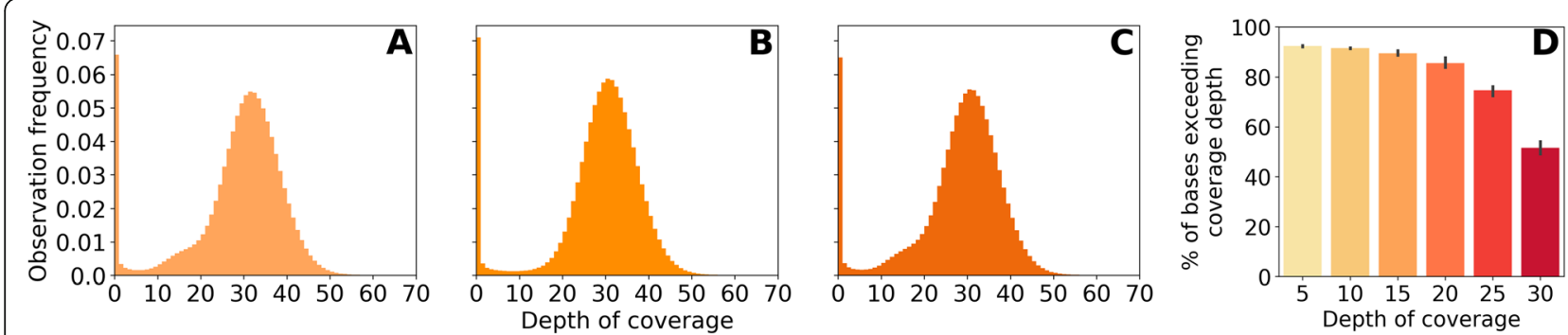

Fig. 1 Whole genome depth of coverage distributions. Metrics for sample_001 (a), sample_002 (b), sample_003 (c) and breadth of coverage for the specified depth thresholds (d) averaged for all three samples are shown with 95\% confidence intervals, $n=3$

genomic regions. Because of that, it was unlikely to detect any unevenness in the WGS-derived discordant genotype calls distribution.

\section{Concordance analysis}

Calculated confusion matrices for all three analyzed DNA samples are shown in Fig. 4, with metrics values presented in Table 3. These values show high overall concordance in genotyping between both WGS and BeadChip techniques. Detected discordant results may have arisen from ambiguous BeadChip genotyping call within multiallelic sites. Nevertheless, all calculated quality metrics values surpassed the recommended thresholds [6].

\section{Genotyping quality metrics distributions}

For all three samples, the distributions of the WGS genotyping metrics were analyzed and compared within the groups formed based on the concordance, variation class and genotype zygosity criteria (data not shown). While differences in mean values were statistically significant (Welch's t-test, $0.05 p$-value threshold) in several groups, distributions themselves exhibited low intergroup divergence. Moreover, for all group comparisons, observed differences were quite small, and their patterns were not uniform, with several groups being insufficiently large, potentially obscuring inter-group dissimilarity. Thus, discordance in genotyping could not be explained by the variance in these quality metrics. When quality metrics were analyzed for the BeadChip calls, some differences in parameters distributions were found. The distinction was remarkable only for the SNV group, as it included the significant amount of all genotyping results and thus could more precisely represent any actual deviations. Inspection of R, Theta and GC scores for both concordant and discordant variants revealed a pattern of discordant variants located close to the borders of the variant clusters (Fig. 5). Importantly, similar clusters are used in the Illumina GenomeStudio software to assign genotypes to variants ( $\mathrm{AA}, \mathrm{AB}$ and $\mathrm{BB}$ ), and, therefore, any genotype lying far from the cluster centre may be mistakenly assigned an irrelevant designation. This explanation for the observed discordance pattern was, however, relevant only to sample_002 and sample_ 003. In sample_001, distribution of discordant variants within the clusters was more or less even. Therefore, we conclude that the observed phenomenon requires further examination. Potential finding of the clustering pattern for discordant calls may then be exploited for quality control.

\section{Sanger sequencing}

The sliding window approach performed on sample_002 resulted in the mapping of 6 regions containing from 1 to 3 variants successfully genotyped on both WGS and BeadChip platforms and exhibiting discordant genotypes between the used platforms. Locations of these regions and encompassed variants are collated in Tables 4 and 5 . A total of 12 discordant variants were selected for validation by Sanger sequencing. These variants were accompanied by 14 concordant variants located closely to discordant variants. These 12 discordant variants included 6 SNVs and 6 INDELs, of which 4 SNVs were of AA vs BB type discordancy, 2 - AA vs AB type, 3 INDE Ls of II vs DD type and the remaining 3 INDELs of DD vs DI (II vs DI) type. The selection was performed based on the following criteria:

1. Selected SNPs were successfully genotyped using both WGS and BeadChip platforms.

Table 2 WGS coverage statistics for the filtered mapped data

\begin{tabular}{llllll}
\hline Sample & Total filtered bases & Mean DOC & \% bases above 10 & \% bases above 20 & \% bases above 30 \\
\hline 001 & 87347116777 & 28.70 & 91.6 & 84.8 & 54.1 \\
002 & 86399503195 & 28.39 & 91.3 & 87.6 & 51.3 \\
003 & 84678940424 & 27.82 & 91.5 & 83.8 & 49.1 \\
\hline
\end{tabular}




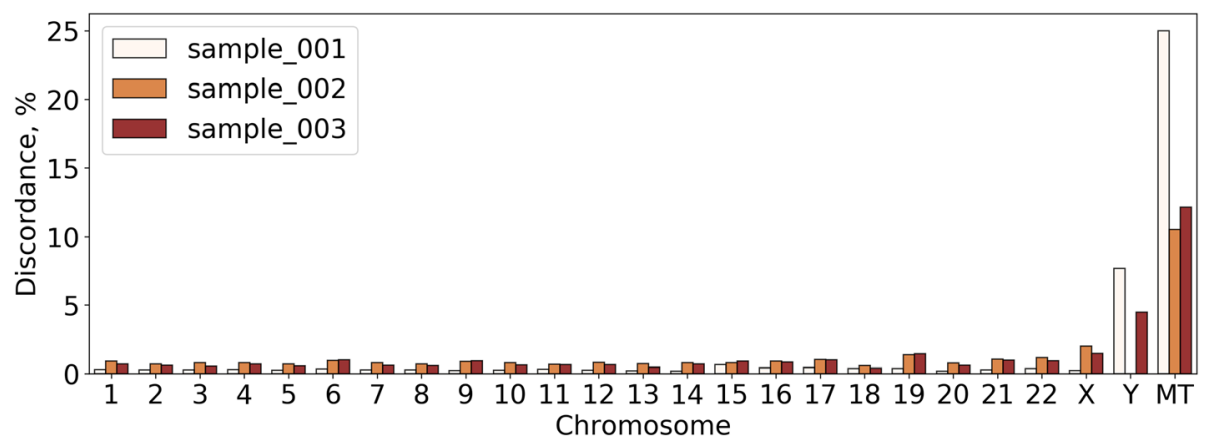

Fig. 2 Fractions of discordant results for three samples. Percentage of discordant results per each chromosome is shown where applicable

2. Selected SNPs are located close to each other within 500 bp window length (reasonable limit of one Sanger read).

3. Specific primers can be selected for these SNPscontaining region (by Primer-BLAST [7]).

The list of designed primers with respective amplification parameters can be found in Table 5. The gDNA of sample_002 was used for amplifying regions of interest (ROIs) in Table 4. All ROIs, except those located on chromosomes 10 and 13, were successfully amplified and Sanger sequenced. The comparison of genotypes obtained via microarray genotyping, whole genome sequencing and Sanger sequencing of the amplified ROIs is shown in Table 6. Sanger-derived genotypes containing only one letter were the ones obtained from only one read. Plus signs denote Sanger genotypes concordant with the WGS-derived genotype, while double plus signs denote concordance of all three genotyping methods. Hashtag represents concordance with the BeadChip-derived genotype, and asterisk - an absence of concordance with any of the tested methods. Sixteen Sanger-resolved diploid genotypes (forward and reverse
Sanger chromatograms covered the variant location) out of the 26 listed in Table 6 were used for confusion matrices calculation using Sanger-derived calls as a "truth" call set, which resulted in WGS and BeadChip precision of 0.81 and 0.5 , respectively, and the accuracy values of 0.87 and 0.61 . Results of Sanger validation may possibly be explained by low complexity or repetitive genomic context which surrounds some of the validated variants, hindering accuracy and precision of either genotyping or read alignment. All the listed variants which were discordant between BeadChip and WGS were plotted (Fig. 5) to reveal possible clustering of discordant variants based on the initial BeadChip genotyping metrics. No clustering of the validated variants was observed. Thus, current analysis did not allow to make any definite conclusions about the clustering of discordant and concordant variants based on their genotyping quality metrics.

\section{Discussion}

Although genotype concordance analysis experiments using different sequencing and genotyping platforms have

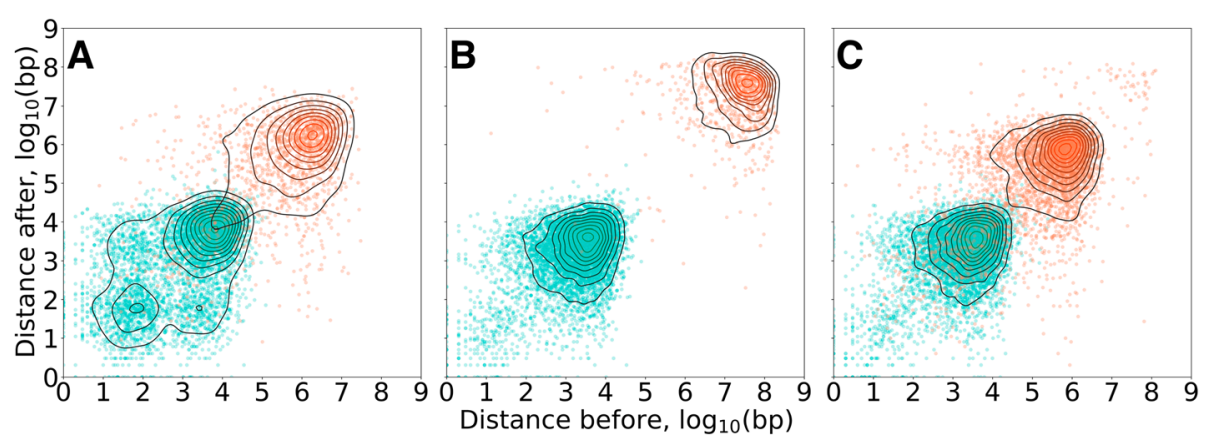

Fig. 3 Distance maps for the analyzed samples. a - sample_001, b - sample_002, c — sample_003, concordant and discordant variants are marked in green and orange, respectively 

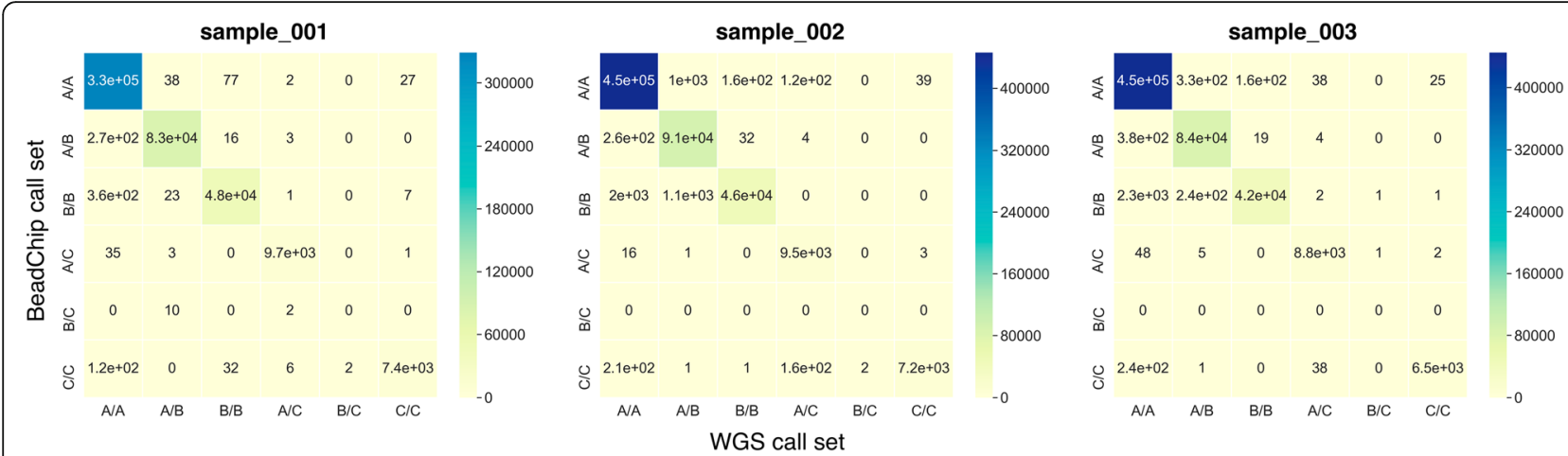

Fig. 4 Confusion matrices calculated for the call sets obtained by WGS and BeadChip. WGS was defined as "true" call set, BeadChip — "test" call set, data is shown for sample_001 (male, chromosomes MT, X, Y were excluded from analysis), sample_002 (female, chromosome MT was excluded from analysis) and sample_003 (male, chromosomes MT, X, Y were excluded from analysis)

been performed previously $[2,8]$, no reports on attempts to explain the observed differences were made. Here we tried to scrutinize the underlying genotyping process proxy such as genotyping quality metrics to find a possible explanation for the discordance pattern. Unfortunately, the genotyping discordance of the observed levels (less than $1 \%$ ) is usually an underestimation, and does not motivate investigations into the nature of the discordant genotype calling. However, we show that discordant genotypes tend to form clusters in a 2-dimensional space, where the most vivid dimensions are the genotyping quality and technical metrics obtained from the BeadChip genotyping pipeline. Therefore, speculation on possibly lower precision of the BeadChip genotyping platform, as compared to WGS-based pipelines, might find a new conceivable basis upon further investigation. Genotype assignment in the array pipelines is based on marker clustering in a 2-dimensional space (clusters $\mathrm{A} / \mathrm{A}, \mathrm{A} / \mathrm{B}, \mathrm{B} / \mathrm{B}$ ), which might happen to be erroneous due to poor cluster separation. This clusterization problem may possibly be solved by incorporating additional dimensions into the analyzed genotyping metrics space, which can be exploited for enhancement of the BeadChip genotyping pipeline upon further investigation and vast Sanger validation of the variants.

\section{Conclusions}

Here we show the presence of some parametric differences in quality metrics of genotyping performed by WGS and BeadChip. This phenomenon warrants comprehensive investigation by combining genotyping metrics produced by WGS and BeadChip pipelines and extracting patterns in the observed discordance. In clusters, Sanger validation should be performed for genotype resolution.

\section{Methods}

\section{Materials}

Three human genomic DNA samples, two males and one female, were selected for this comparison. After collection by Oragene DNA saliva-based collection device (DNA Genotek, Canada), genomic DNA was extracted as per the manufacturer protocol and stored frozen in Tris-EDTA buffer at $-20^{\circ} \mathrm{C}$ until genotyping was performed.

\section{BeadChip genotyping}

Infinium iSelect $24 \times 1$ HTS Custom Beadchip Kit (GSAsharedCUSTOM_20018389_A2) genotyping was performed using $50 \mathrm{ng}$ of genomic DNA. Microarray fluorescence was scanned using the Illumina iScan system, and the genotype calling was executed using the Illumina GenomeStudio Genotyping Module software (Illumina, USA). No imputation was implemented, and only variants successfully genotyped on the specified array were used in the analysis.

\section{Genome sequencing and variant calling}

Whole genome sequencing was performed by MedGenome (CA, USA) using the HiSeq X Ten platform (Illumina, USA) utilizing a 150 base-pair (bp) paired-end

Table 3 Quality metrics of genotyping comparison between BeadChip and WGS call sets

\begin{tabular}{|c|c|c|c|c|c|c|c|}
\hline Sample & $\begin{array}{l}\text { Genotype } \\
\text { concordance }\end{array}$ & $\begin{array}{l}\text { Non-reference genotype } \\
\text { concordance }\end{array}$ & $\begin{array}{l}\text { Non-reference genotype } \\
\text { sensitivity }\end{array}$ & Sensitivity & Precision & Specificity & Accuracy \\
\hline 001 & 0.99783 & 0.99616 & 0.99687 & 0.99783 & 0.99783 & 0.99925 & 0.99928 \\
\hline 002 & 0.99156 & 0.97973 & 0.98773 & 0.99156 & 0.99156 & 0.99696 & 0.99718 \\
\hline 003 & 0.99253 & 0.985 & 0.98402 & 0.99253 & 0.99253 & 0.99742 & 0.9975 \\
\hline
\end{tabular}



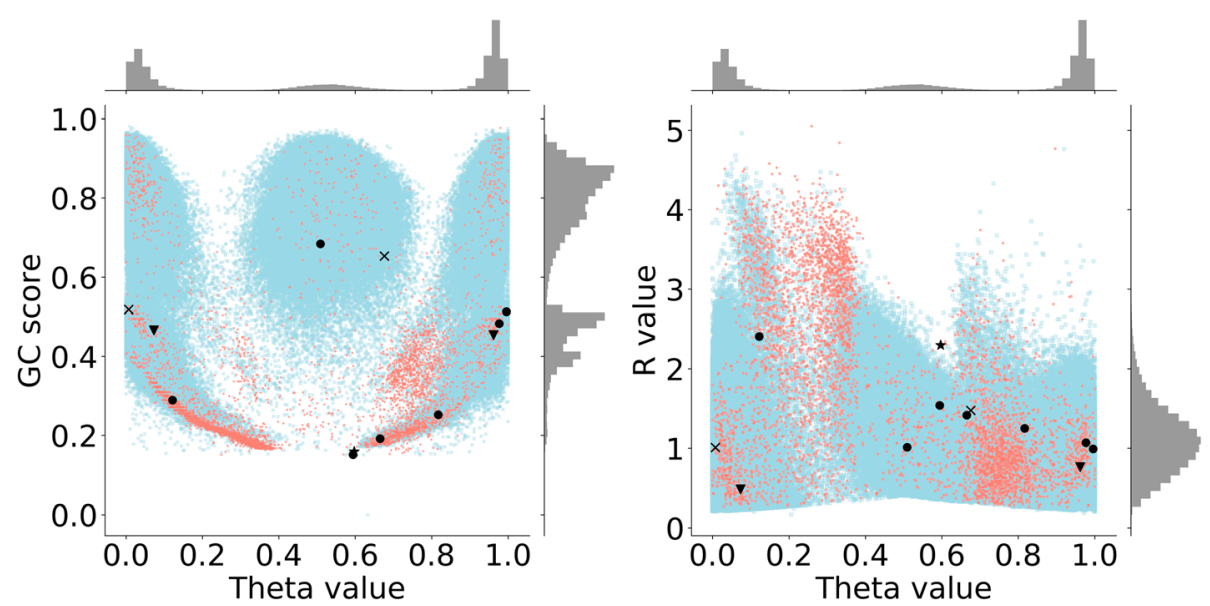

Fig. 5 BeadChip genotyping quality metrics with highlighted Sanger-validated variants. Theta, R, GC Score values for sample_002 are shown; histograms show the corresponding distributions of plotted metrics in a 1-dimensional space; concordant and discordant variants are marked in blue and orange, respectively; genotypes which are not consistent with Sanger sequencing in both WGS and BeadChip results are marked with a star, matches between Sanger and BeadChip are marked with triangles, matches between Sanger and WGS are marked with circles, variants which were not successfully genotyped by Sanger are marked with crosses

protocol. The raw FASTQ files were evaluated using FastQC software (Babraham Institute, UK). A proprietary WGS data processing pipeline was designed to process NGS data in automated mode from raw FASTQ files to finalized genotyping data. The pipeline implemented adapter trimming using Trimmomatic [9], BWA MEM [10] alignment to the GRCh38 build of the human genome, duplicate reads marking by Picard (Broad Institute, USA), filtering of the resulting SAM/BAM files via SAMtools $[11,12]$. It also utilized GATK tools (Broad Institute, USA) to perform base quality score recalibration (BQSR), genotype calling and variant quality score recalibration (VQSR). GATK VariantAnnotator and
BCFtools were employed to perform variant annotation. The pipeline implemented several hard filtering procedures resulting in a final VCF output with all variant and non-variant sites which passed quality control of the filtering step. The pipeline was designed in compliance with GATK Best Practices recommendations for NGS data processing. Single nucleotide variants $(\mathrm{SNVs})$ and indels were called using GATK HaplotypeCaller with subsequent VQSR using a threshold of 99.9, HapMap 3.3, 1000G Omni 2.5, 1000G Phase 1 High Confidence and dbSNP build 151 training sets for SNV mode and Mills and 1000G Gold Standard Indels with dbSNP build 151 training sets for INDEL mode.

Table 4 The chosen regions for validation by Sanger sequencing for sample_002

\begin{tabular}{|c|c|c|c|c|c|c|c|c|}
\hline \multirow[t]{2}{*}{ Chromosome } & \multirow[t]{2}{*}{ Start } & \multirow[t]{2}{*}{ End } & \multirow{2}{*}{$\begin{array}{l}\text { INDE } \\
\text { LS }\end{array}$} & \multirow[t]{2}{*}{ SNVs } & \multirow[t]{2}{*}{ Total } & \multicolumn{2}{|l|}{ Genotypes } & \multirow[t]{2}{*}{ Vartype } \\
\hline & & & & & & BeadChip & WGS & \\
\hline \multirow[t]{3}{*}{1} & \multirow[t]{3}{*}{109711850} & \multirow[t]{3}{*}{109712150} & \multirow[t]{3}{*}{0} & \multirow[t]{3}{*}{3} & \multirow[t]{3}{*}{3} & TG & GG & \multirow[t]{3}{*}{3 SNVs } \\
\hline & & & & & & TC & $\pi$ & \\
\hline & & & & & & $C G$ & GG & \\
\hline 1 & 109759300 & 109759400 & 1 & 0 & 1 & DD & $\|$ & 1 INDEL \\
\hline 2 & 85663600 & 85663700 & 1 & 0 & 1 & DI & $\|$ & 1 INDEL \\
\hline 2 & 233671750 & 233671850 & 1 & 0 & 1 & $\|$ & $\mathrm{DI}$ & $1 \mathrm{INDEL}$ \\
\hline \multirow[t]{3}{*}{6} & \multirow[t]{3}{*}{160246180} & \multirow[t]{3}{*}{160246690} & \multirow[t]{3}{*}{0} & \multirow[t]{3}{*}{3} & \multirow[t]{3}{*}{3} & GG & AA & \multirow[t]{3}{*}{3 SNVS } \\
\hline & & & & & & $\pi$ & $\mathrm{CC}$ & \\
\hline & & & & & & GG & AA & \\
\hline 10 & 101010500 & 101010600 & 1 & 0 & 1 & DD & $\|$ & 1 INDEL \\
\hline 13 & 49792500 & 49792600 & 1 & 0 & 1 & DD & $\mathrm{Dl}$ & 1 INDEL \\
\hline 22 & 37973800 & 37974170 & 1 & 0 & 1 & DD & $\|$ & 1 INDEL \\
\hline
\end{tabular}


Table 5 The designed primers for amplification and sequencing of the chosen regions

\begin{tabular}{|c|c|c|c|c|c|c|c|c|c|c|c|}
\hline Chromosome & Orientation & Name & Sequence $5{ }^{\prime} 3^{\prime}$ & Length & $\mathrm{Tm}$ & GC & Start & End & $\begin{array}{l}\text { Amplifying } \\
\text { length }\end{array}$ & $\begin{array}{l}\mathrm{N} \\
\text { mismatches }\end{array}$ & $\begin{array}{l}\text { Mismatch } \\
\text { type }\end{array}$ \\
\hline 1 & $\mathrm{~F}$ & $\begin{array}{l}{ }_{\text {chr1_- }} \\
\text { reg1_f }\end{array}$ & $\begin{array}{l}\text { AAGCCCTCGGAGTAGC } \\
\text { TाTC }\end{array}$ & 20 & 59.46 & 55 & 109711755 & 109711774 & 397 & 3 & $3 \mathrm{SNV}$ \\
\hline 1 & $\mathrm{R}$ & $\begin{array}{l}\text { Chr1_- } \\
\text { reg1_R }\end{array}$ & $\begin{array}{l}\text { GGCTGGAATCAATAAG } \\
\text { CCCC }\end{array}$ & 20 & 58.67 & 55 & 109712151 & 109712132 & 397 & 3 & $3 \mathrm{SNV}$ \\
\hline 1 & $\mathrm{~F}$ & $\begin{array}{l}\text { Chr1_- }_{\text {reg2_F }} \\
\end{array}$ & $\begin{array}{l}\text { TGATGGACAG } \\
\text { GATGGAGTTGTAG }\end{array}$ & 23 & 59.55 & 47.83 & 109759300 & 109759322 & 85 & 1 & 1 INDEL \\
\hline 1 & R & $\begin{array}{l}\text { chr1_- } \\
\text { reg2_R }\end{array}$ & $\begin{array}{l}\text { GAGCTGGACTCTTACC } \\
\text { GCCTA }\end{array}$ & 21 & 61.02 & 57.14 & 109759384 & 109759364 & 85 & 1 & 1 INDEL \\
\hline 2 & $\mathrm{~F}$ & $\begin{array}{l}\text { chr2__ } \\
\text { reg1_F }\end{array}$ & $\begin{array}{l}\text { GTGGTCACGGACAT } \\
\text { GCAGA }\end{array}$ & 19 & 60.01 & 57.89 & 85663428 & 85663446 & 344 & 1 & 1 INDEL \\
\hline 2 & $\mathrm{R}$ & $\begin{array}{l}\text { chr2_- } \\
\text { reg1_R }\end{array}$ & $\begin{array}{l}\text { CTGAGCGCTACTCCGT } \\
\text { CATC }\end{array}$ & 20 & 60.32 & 60.00 & 85663771 & 85663752 & 344 & 1 & 1 INDEL \\
\hline 2 & $\mathrm{~F}$ & $\begin{array}{l}\text { chr2_- } \\
\text { reg2_F }\end{array}$ & $\begin{array}{l}\text { CTCTGGACAGAGAG } \\
\text { TATTGGTTG }\end{array}$ & 24 & 58.83 & 45.83 & 233671510 & 233671533 & 370 & 1 & 1 INDEL \\
\hline 2 & R & $\begin{array}{l}\text { chr2_- } \\
\text { reg2_R }\end{array}$ & $\begin{array}{l}\text { AGGTGGGAGAAATA } \\
\text { CCAGCAC }\end{array}$ & 21 & 59.72 & 52.38 & 233671879 & 233671859 & 370 & 1 & 1 INDEL \\
\hline 6 & $\mathrm{~F}$ & chr6_F & $\begin{array}{l}\text { TTAAGAAGG } \\
\text { CAGGGGATTGCT }\end{array}$ & 22 & 59.96 & 45.45 & 160246431 & 160246452 & 490 & 3 & 3 SNV \\
\hline 6 & R & chr6_R & $\begin{array}{l}\text { GCAACTTAAGCCTTCA } \\
\text { CCAGT }\end{array}$ & 21 & 58.77 & 47.62 & 160246920 & 160246900 & 490 & 3 & 3 SNV \\
\hline 10 & $\mathrm{~F}$ & Chr10_F & $\begin{array}{l}\text { CCTGGAGACTTGCC } \\
\text { TTGACC }\end{array}$ & 20 & 60.32 & 60 & 101010511 & 101010530 & 233 & 1 & 1 INDEL \\
\hline 10 & $\mathrm{R}$ & Chr10_R & $\begin{array}{l}\text { CCTCTACAAGACGTGC } \\
\text { CAGT }\end{array}$ & 20 & 59.40 & 55 & 101010743 & 101010724 & 233 & 1 & 1 INDEL \\
\hline 13 & $F$ & Chr13_F & $\begin{array}{l}\text { AAGCTCTTGATGCG } \\
\text { GTGGTT }\end{array}$ & 20 & 60.25 & 50 & 49792463 & 49792482 & 330 & 1 & 1 INDEL \\
\hline 13 & $\mathrm{R}$ & Chr13_R & $\begin{array}{l}\text { CACGTATAGCCCGG } \\
\text { CGAA }\end{array}$ & 18 & 59.59 & 61.11 & 49792792 & 49792775 & 330 & 1 & 1 INDEL \\
\hline 22 & $F$ & chr22_F & $\begin{array}{l}\text { CGAGGGCCCCATATAG } \\
\text { GAGA }\end{array}$ & 20 & 59.96 & 60 & 37973603 & 37973622 & 408 & 1 & 1 INDEL \\
\hline 22 & $\mathrm{R}$ & chr22_R & $\begin{array}{l}\text { GAGTTGGACCAGTACC } \\
\text { TGCC }\end{array}$ & 20 & 60.04 & 60 & 37974010 & 37973991 & 408 & 1 & 1 INDEL \\
\hline
\end{tabular}

\section{Quality metrics and validation statistics}

Concordance analysis implied calculation of specificity, sensitivity, precision, accuracy, genotype concordance, non-reference genotype concordance and non-reference sensitivity of genotyping between BeadChip and WGS. The analysis implemented confusion matrices calculation (Fig. 6). As there is no baseline "truth" when a WGS call set is compared with a BeadChip one, no method can be described as "comparator". Because of this, each call set within each pair was treated as alternating "truth" or "test" call set, followed by the averaging of obtained statistics. Filling those matrices with observed numbers of class counts was followed by dimensionality reduction, which implied leaving one class in both call sets intact and combining the counts in all other classes (Fig. 6). The above-specified quality metrics for the initial and reduced matrices were calculated, as shown in Fig. 7 [2] with amendments. As each non- reduced matrix produced 6 submatrices, the calculated sensitivity and precision value for each submatrix was weighted by the fraction of analyzed elements in a nonreduced matrix (i.e., each calculated metric was then multiplied by the ratio of orange-outlined elements to all elements in the reduced matrix in Fig. 7). Such an approach was only necessary for precision and sensitivity metrics due to the fact that these metrics were calculated on non-overlapping subsets of the initial matrix and their values depended on the size of those subsets, given uneven marker distribution in the initial matrix. Other used metrics (accuracy, specificity) were calculated as mean values between 6 produced metric values (excluding NANs, which originated from calculations involving division by zero).

All concordant and discordant genotyped variants were analyzed for genotyping quality metrics provided in the VCF files and GenomeStudio report files for WGS 
Table 6 Sanger validation results for sample_002

\begin{tabular}{|c|c|c|c|c|c|}
\hline rsID & Chromosome & Position (GRCh38) & BeadChip genotype & WGS genotype & Sanger genotype \\
\hline rs12731384 & 1 & 109711898 & $\mathrm{TG}$ & GG & $\pi$ \\
\hline rs1633365 c & 1 & 109711919 & TC & $\pi$ & $\mathrm{TC}$ \\
\hline rs58877308 ${ }^{a}$ & 1 & 109712113 & CG & GG & GG \\
\hline rs138687644 & 1 & 109759369 & GG & GG & $\mathrm{G}_{-}$ \\
\hline rs202018423 b & 1 & 109759339 & $\mathrm{CC}$ & CC & $\mathrm{CC}$ \\
\hline rs757293027 & 1 & 109759384 & $\mathrm{CC}$ & CC & $C_{-}$ \\
\hline rs760279355 b & 1 & 109759346 & $\mathrm{CC}$ & CC & $\mathrm{CC}$ \\
\hline rs767809812 & 1 & 109759359 & DD & $\|$ & $1-$ \\
\hline rs79394341 & 1 & 109759361 & GG & GG & $\mathrm{G}_{-}$ \\
\hline rs786205634 & 2 & 85663647 & DI & $\|$ & $\|$ \\
\hline rs3832043 & 2 & 233671807 & $\|$ & $\mathrm{Dl}$ & $\mathrm{DD}$ \\
\hline rs144484152 & 6 & 160246683 & GG & AA & AA \\
\hline rs149262397 b & 6 & 160246574 & $\mathrm{CC}$ & CC & $\mathrm{CC}$ \\
\hline rs316022 & 6 & 160246568 & GG & AA & AA \\
\hline rs529525717 b & 6 & 160246473 & GG & GG & GG \\
\hline rs537568133 & 6 & 160246590 & $\pi$ & $C C$ & $\mathrm{CC}$ \\
\hline rs549969754 & 6 & 160246607 & $\mathrm{CC}$ & CC & CC \\
\hline rs555024471 & 6 & 160246297 & GG & GG & G- \\
\hline rs563560445 b & 6 & 160246467 & CC & CC & CC \\
\hline rs563829592 & 6 & 160246443 & GG & GG & G- \\
\hline rs577352795 & 6 & 160246460 & $\pi$ & $\pi$ & T- \\
\hline rs757852385 & 6 & 160246688 & AA & AA & AA \\
\hline rs759566284 & 6 & 160246458 & GG & GG & G- \\
\hline rs200896335 & 10 & 101010536 & DD & $\|$ & NA \\
\hline rs753420953 & 13 & 49792587 & DD & $\mathrm{DI}$ & NA \\
\hline rs397515367 & 22 & 37973817 & DD & $\|$ & $\|$ \\
\hline
\end{tabular}

${ }^{a}$ concordance between Sanger and WGS genotypes

${ }^{b}$ concordance between all three genotyping methods

c concordance between Sanger and BeadChip genotypes

dabsence of concordance between any of the methods

and BeadChip genotyping, respectively. The following quality metrics obtained via WGS genotyping pipeline were analyzed:

1. DP variant read depth at a particular position for a particular sample.

2. QUAL Phred-scaled quality score for the assertion made in ALT. i.e., $-10 \log _{10}\left(P_{\text {call }}\right.$ in ALT is wrong $)$, if ALT is "." (no variant) then this is $-10 \log _{10}\left(P_{\text {variant }}\right)$, and if ALT is not "." this is $-10 \log _{10}\left(P_{\text {no variant }}\right)$.

3. RGQ unconditional reference genotype confidence, encoded as a Phred quality $-10 \log _{10}\left(P_{\text {genotype call is }}\right.$ wrong).

4. GQ conditional genotype quality, encoded as a Phred quality $-10 \log _{10}\left(P_{\text {genotype call is wrong }}\right)$, conditioned on the site is variant.
The following metrics were extracted from corresponding GenomeStudio reports:

1. GC score GenCall score, a quality metric that indicates the reliability of each genotype call. The GenCall Score is a value between 0 and 1 assigned to every called genotype. Genotypes with lower GenCall scores are located further from the centre of a cluster and have lower reliability.

2. GT score GenTrain score, a quality metric that indicates how well the samples clustered for a locus.

3. Cluster Sep cluster separation score.

4. Theta - the normalized Theta-value of the SNP for the sample. 


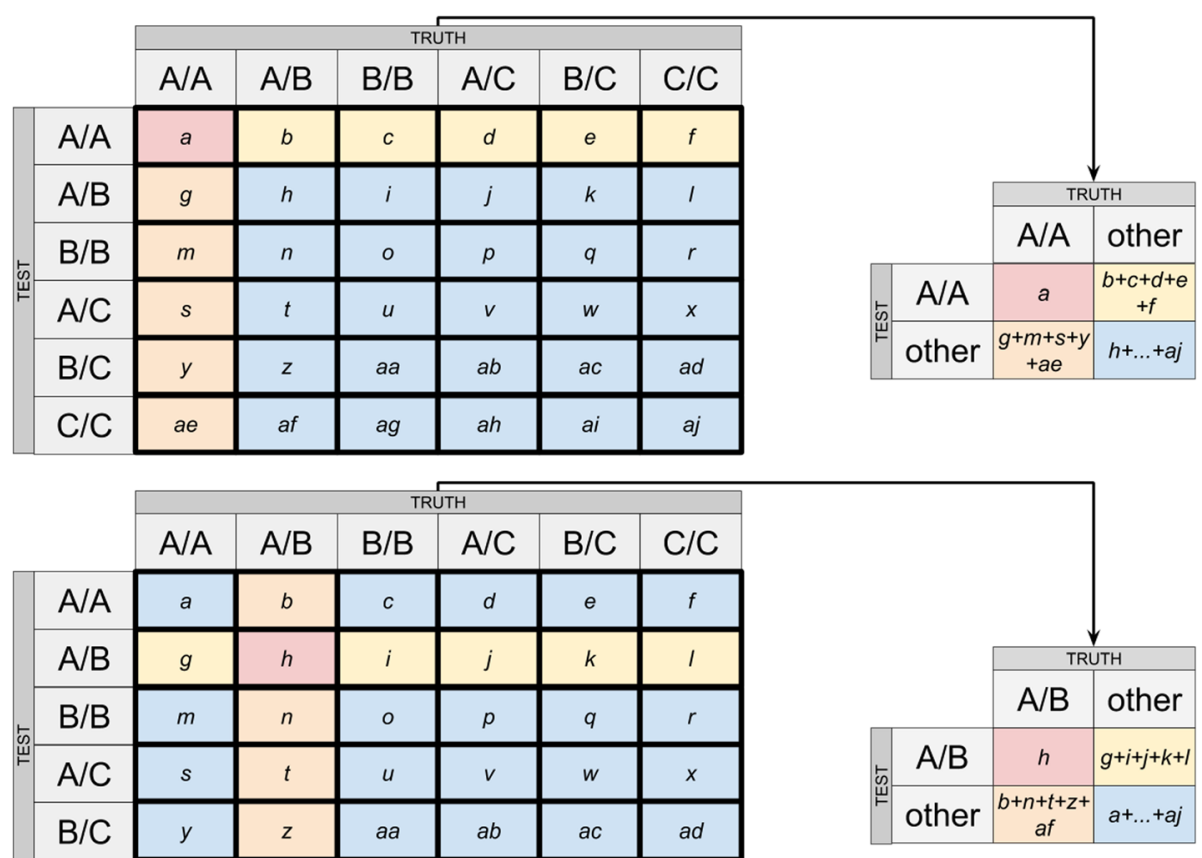

Fig. 6 Example calculation of confusion matrices. The shown dimensionality reduction is used for accuracy and other metrics calculation; $a$, b, $C$, $d, \ldots$, ai, aj — sample counts of each class; A/A, A/B, B/B, A/C, B/C, C/C - diploid genotypes observed in data; A - reference allele, B and C alternative alleles. TRUTH — a call set produced by an orthogonal method (comparator), TEST — a call set produced by a test method

5. $\mathrm{R}$ - the normalized R-value of the SNP for the sample.

6. $\mathrm{X}-$ the normalized intensity of the A allele.

7. $\mathrm{Y}-$ the normalized intensity of the B allele.
8. B Allele Freq B allele theta value of the SNP for the sample, relative to the cluster positions. This value is normalized so that it is zero if theta is less than or equal to the AA cluster's theta mean, 0.5 if it is

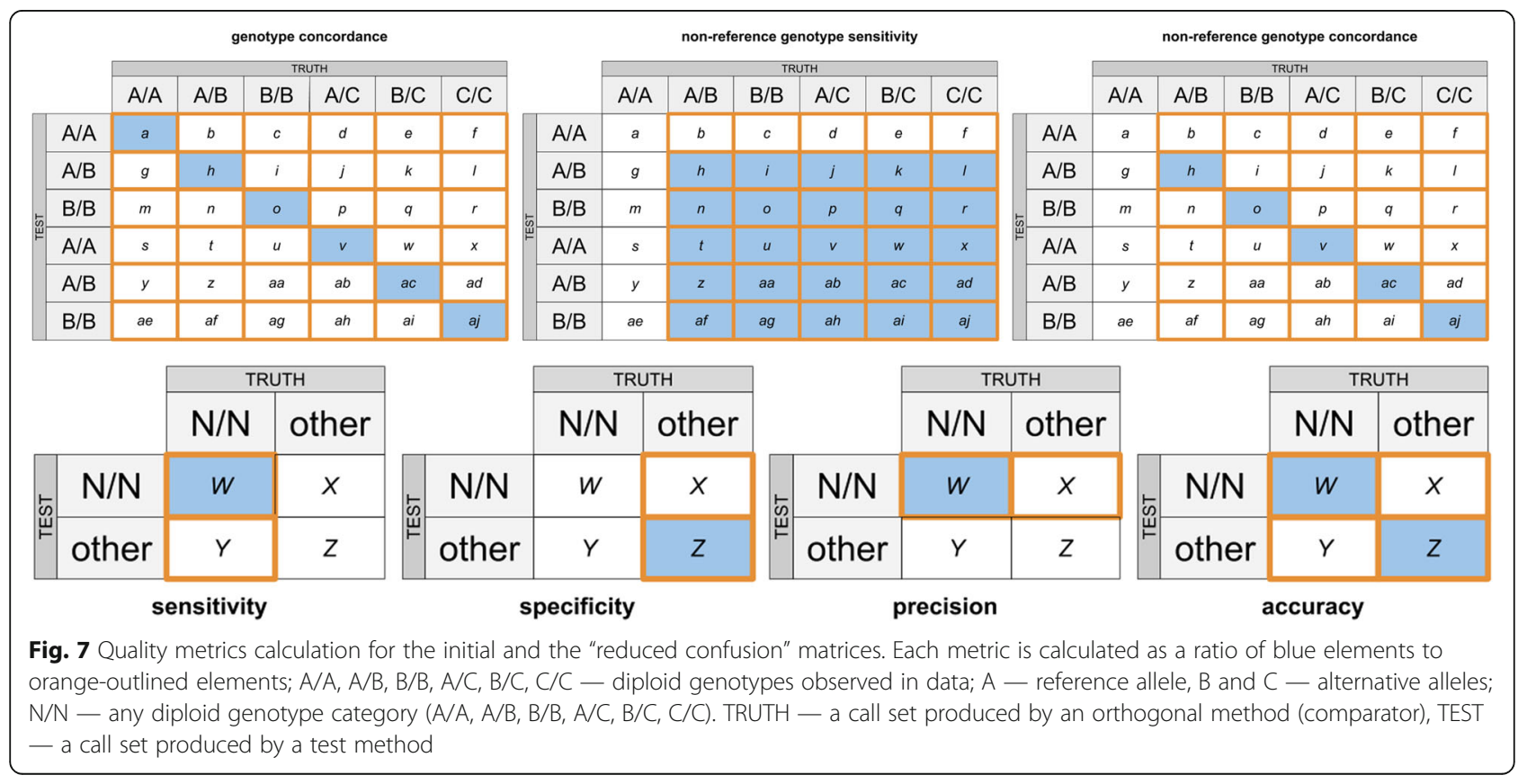


equal to the $A B$ cluster's theta mean, or 1 if it is equal to or greater than the BB cluster's theta mean. B Allele Freq is linearly interpolated between 0 and 1.

\section{Variant selection for sanger sequencing}

An intersection of WGS- and array-genotyped markers which exhibited discordant calls between the two platforms was used for selection. A sliding window approach was utilized to find regions spanning no more than 500 bp (Sanger sequencing reasonable read length limit) and encompassing as many discordant variants from the selected set as possible. All primers for PCR amplification were designed using NCBI Primer-BLAST suite [7] with default parameters (except the melting temperature limits of $58-62{ }^{\circ} \mathrm{C}$ ) and the human genome reference sequence for BLAST. PCR product lengths and primer lengths were manually optimized to find the most suitable unique match. Primer synthesis, amplification and Sanger sequencing was performed by Evrogen (Russia, Moscow). Sanger chromatograms were visualized and analyzed using 4Peaks software (Nucleobytes, The Netherlands) and CodonCode Aligner (CodonCode Corp., USA) with default trimming and quality filtering parameters.

\section{Supplementary information}

Supplementary information accompanies this paper at https://doi.org/10. 1186/s12864-020-06919-x.

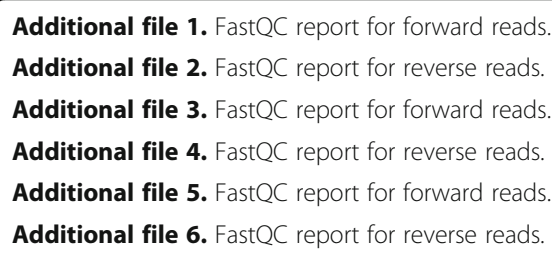

\section{Abbreviations}

WGS: Whole genome sequencing; WES: Whole exome sequencing; NGS: Next-generation sequencing; PCR: Polymerase chain reaction; DOC: Depth of coverage; BOC: Breadth of coverage; GATK: Genome analysis toolkit; SNP: Single nucleotide polymorphism; INDEL: Insertion-deletion variation; EDTA: Ethylenediaminetetraacetic acid; BQSR: Base quality score recalibration; VQSR: Variant quality score recalibration

\section{Acknowledgements}

We thank the members of Atlas Biomed Research and Development department for discussion and critical analysis of data processing and statistical approaches.

\section{About this supplement}

This article has been published as part of BMC Genomics Volume 21 Supplement 7, 2020: Selected Topics in "Systems Biology and Bioinformatics" - 2019: genomics. The full contents of the supplement are available online at https://bmcgenomics.biomedcentral.com/articles/supplements/volume-21supplement-7.

\section{Authors' contributions}

The data was provided by Atlas Biomed. KAD, AVB and DAN designed the experiments and conducted the research, KAD designed Sanger sequencing experiments, performed bioinformatical analysis of WGS data, DAN analyzed BeadChip data, KAD drafted the work, DAN, AVB and SVM revised the work, AVB and SVM contributed the majority of the writing with input from all authors. All authors have read and approved the submitted version.

\section{Funding}

Publication costs have been funded by Atlas Biomed. Atlas Biomed was not involved in the design of the study, collection, analysis or interpretation of data, in writing the manuscript, and in the decision to publish the results.

\section{Availability of data and materials \\ The datasets generated and/or analysed during the current study are not publicly available due Atlas Biomed personal data privacy policy but are available from the corresponding author on reasonable request.}

\section{Ethics approval and consent to participate}

The research was approved by the local ethics committee of the Atlas Medical Center, LLC. The project was conducted in accordance with the principles expressed in the Declaration of Helsinki. All participants have signed the informed consent forms before entering the study.

\section{Consent for publication}

Not applicable.

\section{Competing interests}

$K A D, D A N$ and SVM are employees of Atlas Biomed. AVB is an employee of George Mason University, Virginia, USA. Atlas Biomed offers genotyping and genome sequencing services. The authors declare no other competing interests.

\section{Author details}

${ }^{1}$ Atlas Biomed Group Limited, Tintagel House, 92 Albert Embankment, Lambeth, London SE1 7TY, UK. 'Skolkovo Institute of Science and Technology, Bolshoy Boulevard 30, bld. 1, 121205 Moscow, Russia. ${ }^{3}$ School of Systems Biology, George Mason University, 10900 University Blvd, Fairfax, VA 22030, USA. ${ }^{4}$ Research Center for Medical Genetics, Moskvorechye St., 1, 115478 Moscow, Russia.

Received: 14 July 2020 Accepted: 17 July 2020

Published: 10 September 2020

\section{References}

1. Gargis AS, Kalman L, Berry MW, Bick DP, Dimmock DP, Hambuch T, Lu F, Lyon E, Voelkerding KV, Zehnbauer BA, Agarwala R. Assuring the quality of next-generation sequencing in clinical laboratory practice. Nat Biotechnol. 2012;30(11):1033.

2. Linderman MD, Brandt T, Edelmann L, Jabado O, Kasai $Y$, Kornreich R, Mahajan M, Shah H, Kasarskis A, Schadt EE. Analytical validation of whole exome and whole genome sequencing for clinical applications. BMC Med Genet. 2014:7(1):20.

3. Steemers FJ, Gunderson KL. Whole genome genotyping technologies on the BeadArray ${ }^{\mathrm{TM}}$ platform. Biotechnol J. 2007;2(1):41-9.

4. Beck TF, Mullikin JC, Biesecker LG, Comparative Sequencing Program NISC Systematic evaluation of sanger validation of next-generation sequencing variants. Clin Chem. 2016:62(4):647-54.

5. Broad Institute. GATK Tools; (version 3.8). Available from: http://github.com/ broadinstitute/gatk/. Accessed 5 Mar 2018.

6. Rehm HL, Bale SJ, Bayrak-Toydemir P, Berg JS, Brown KK, Deignan JL, Friez MJ, Funke BH, Hegde MR, Lyon E. ACMG clinical laboratory standards for next-generation sequencing. Genet Med. 2013;15(9):733.

7. Ye J, Coulouris G, Zaretskaya I, Cutcutache I, Rozen S, Madden TL. PrimerBLAST: a tool to design target-specific primers for polymerase chain reaction. BMC Bioinform. 2012;13(1):134

8. Wang Z, Liu X, Yang BZ, Gelernter J. The role and challenges of exome sequencing in studies of human diseases. Front Genet. 2013;4:160.

9. Bolger AM, Lohse M, Usadel B. Trimmomatic: a flexible trimmer for Illumina sequence data. Bioinformatics. 2014;30(15):2114-20.

10. Li H. Aligning sequence reads, clone sequences and assembly contigs with BWA-MEM. arXiv preprint arXiv:1303.3997; 2013. 
11. Li H, Handsaker B, Wysoker A, Fennell T, Ruan J, Homer N, Marth G, Abecasis $G$, Durbin R. The sequence alignment/map format and SAMtools. Bioinformatics. 2009;25(16):2078-9.

12. Li H. A statistical framework for SNP calling, mutation discovery, association mapping and population genetical parameter estimation from sequencing data. Bioinformatics. 2011;27(21):2987-93.

\section{Publisher's Note}

Springer Nature remains neutral with regard to jurisdictional claims in published maps and institutional affiliations.

- fast, convenient online submission

- thorough peer review by experienced researchers in your field

- rapid publication on acceptance

- support for research data, including large and complex data types

- gold Open Access which fosters wider collaboration and increased citations

- maximum visibility for your research: over $100 \mathrm{M}$ website views per year

At $\mathrm{BMC}$, research is always in progress. 